\title{
CAMBIOS EN LOS PERFILES LABORALES EN LA REPÚBLICA DOMINICANA COMO RESULTADO DE LA PANDEMIA
}

\section{Changes in labor profiles in the Dominican Republic as a result of the pandemic}

\section{Fernando Barrero}

Vicerrector académico y de investigación de Barna

Management School, República Dominicana.

ORCID: 0000-0002-7826-890X

Correo-e: fernando.barrero@barna.edu.do
Javier Villamizar

Investigador Asociado de Barna Management School. ORCID: 0000-0001-6083-2835

Correo-e: javierfvillamizarf@yahoo.com

Recibido: $x x x x x \bullet$ Aprobado: $x x x$

Cómo citar: Villamizar, J., \& Barrero, F. (2021). Cambios en los perfiles laborales en la República Dominicana como resultado de la pandemia. Ciencia y Sociedad, 46(2), 31-45. DOI: https://doi.org/10.22206/cys.2021.v46i2.pp31-45

\section{Resumen}

Este estudio permite cuantificar los cambios que se han presentado como resultado de la pandemia con relación al perfil requerido de trabajadores en la República Dominicana. La metodología aplicada fue el análisis de la demanda de personal solicitada por las cinco bolsas o agencias de empleo con mayor número de avisos web de requerimientos de empleados en el país. La mayor parte de puestos vacantes actuales se concentra en técnicos profesionales de nivel medio, reduciéndose la proporción de solicitudes de personal para labores de administración, ventas y servicios. Este comportamiento sugiere que se disminuyeron las vacantes disponibles en los empleos asociados a la cadena de valor del sector turístico y de comercio, los más golpeados por la pandemia. Adicionalmente, la mayor proporción de las empresas continúa solicitando personal en edades de 25 a 45 ańos y hombres. Por otra parte, en el último año, han exigido mayores habilidades blandas, tales como trabajo en equipo, destrezas en comunicación, enfoque a resultados, responsabilidad y liderazgo, así como el dominio de competencias duras como TICs y del idioma inglés. Lo anterior abre un amplio abanico de oportunidades de desarrollo de programas y ajustes de los modelos educativos, como también la adopción de políticas para incentivar el empleo en los grupos poblacionales más vulnerables.

Palabras clave: habilidades blandas; competencias duras; modelo educativo; pandemia.

\begin{abstract}
This study quantified the changes that have occurred in relation to the required job profiles in the Dominican Republic after pandemic. The methodology used was the analysis of the personnel demand requested by the five employment platforms or agencies with the highest number of web notices of employee requirements in the country. Most of the current vacancies are concentrated in professional mid-level technicians, decreasing the proportion of requests for personnel for administration, sales and service tasks declining compared before pandemic. This behavior suggests that the vacancies available in the jobs associated with the tourism value chain and commerce sector decreased, the ones hardest hit by the pandemic. Additionally, most of the companies continue to request staff of men between the ages of 25 and 45 . In 2021, more companies have demanded soft skills, such as teamwork, communication skills, focus on results, responsibility and leadership. On the other hand, requests for hard skills have increased, such as ICT and the English language. The above opens a wide range of opportunities for program development and adjustments of educational models (curriculum), as well as the adoption of policies to encourage employment in the most vulnerable population groups in the country.
\end{abstract}

Keywords: soft skills; hard skills; educational model; pandemic. 


\section{Introducción}

En el año 2020, a nivel mundial se perdieron 114 millones de puestos de trabajo en relación con 2019. En términos relativos, las pérdidas de empleo fueron más altas para las mujeres $(5,0 \%)$ que, para los hombres. De manera equivalente, una mayor proporción de trabajadores jóvenes $(8,7 \%)$ perdieron sus empleos comparada con los mayores. El desempleo mundial aumentó en 33 millones en 2020, y la tasa de desempleo aumentó en 1,1 puntos porcentuales, llegando hasta el 6,5 \% (International Labour Organization, 2021).

Durante el año 2020, los mercados laborales en América Latina y el Caribe retrocedieron al menos 10 años y la problemática está lejos de terminar, debido al negativo impacto sin precedentes de la crisis de COVID-19. De manera equivalente, el desempleo se ha reducido en la población de 15 a 24 años y en las mujeres (Organización Internacional del Trabajo, 2020), grupos poblacionales que generalmente han sido los de menor ocupación laboral en la República Dominicana (Barna Management School y COPARDOM, 2020; Organización Internacional del Trabajo, 2019). Por otra parte, el sector del turismo presentó la mayor contracción económica y, por ende, la mayor caída en empleabilidad en la región, aspecto que ha sido similar en la República Dominicana, donde el desempleo nacional pasó del $5.7 \%$ al $9.5 \%$ en el período de marzo a septiembre de 2020 (Universidad Autónoma de Santo Domingo, 2020), y la contribución de la actividad económica de los hoteles, bares y restaurantes al PIB total pasó del $14.4 \%$ al $5.6 \%$ (Banco Central, 2021) en ese mismo período (abril a septiembre). En un reciente reporte del Fondo Monetario Internacional se menciona que, "en Latinoamérica, la crisis del COVID-19 ha repercutido desproporcionadamente en el empleo, y las pérdidas se han concentrado sobre todo en las mujeres, los jóvenes y los trabajadores informales y menos cualificados" (Banca y Negocios, 2021), aspecto que comparte la República Dominicana, donde la Organización Internacional del Trabajo encontró que los grupos más vulnerables son precisamente las mujeres, los mayores de 45 años y los más jóvenes (Organización Internacional del Trabajo, 2020), lo que exacerba el problema en estos grupos poblacionales, ya golpeados históricamente por la carencia de suficientes oportunidades laborales. No obstante, en muchos países, sectores y grupos ocupacionales vinculados con la salud y tecnología de la información han crecido exponencialmente (Forbes, 2020; LinkedIn, 2020), sugiriendo que el sector primario y aquellos relacionados a mercados de la Cuarta Revolución Industrial (CRI) son los menos afectados a nivel económico y social.

De manera complementaria, al analizar las asimetrías entre la oferta y la demanda del trabajo en la República Dominicana, se ha encontrado que la edad y la formación son los factores que mayor brecha presentan en el país, puesto que la mayoría de las empresas demandan personal entre los 25 y 45 años, con formación en educación superior, en tanto, la mayor proporción de desocupados con relación a la población se encuentra entre los menores de 25 años y mayores de 45 años, así como en los trabajadores con estudios de secundaria (Barna Management School y COPARDOM, 2020).

Por lo anterior, se hace necesario conocer más detalladamente las tendencias y cambios en el mercado del trabajo en la República Dominicana generadas durante la pandemia, tanto a nivel de perfil demográfico como de formación, experiencia, perfil ocupacional y de competencias, de tal forma que los actores participantes del ecosistema del trabajo logren identificar oportunidades y desarrollar soluciones de valor agregado para el desarrollo de iniciativas que permitan mejorar la empleabilidad, reducir las brechas sociales y de edades que ha conllevado el disruptivo auge tecnológico, optimizar el trabajo decente y sembrar el camino para que los futuros profesionales logren 
capitalizar al máximo las bondades que ofrece la Cuarta Revolución Industrial (CRI), participando competitivamente en procesos de selección de los perfiles emergentes.

\section{Bondades y retos de la Cuarta Revolución Indus- trial validadas durante la pandemia}

La Cuarta Revolución Industrial (CRI o Industria 4.0) no se ha detenido a pesar de la pandemia mundial. Por el contrario, el COVID-19 solo ha acelerado la adopción de la Industria 4.0, lo que ha llevado a empresas de todas las industrias a utilizar la tecnología de Internet de las cosas (IoT, por sus siglas en inglés) y Big Data (gran volumen de datos) de una manera más sofisticada. Por su parte, la inteligencia artificial (IA) y la robótica ya no son ciencia ficción. Las empresas deben adaptarse rápidamente a esta realidad. La clave para seguir siendo competitivo es garantizar que se vuelva a capacitar a la fuerza laboral existente y que las generaciones futuras estén equipadas con las habilidades adecuadas para tener éxito (Qlik, 2020). En el contexto actual de la pandemia, las técnicas de IA como el aprendizaje automático (ML), la optimización y el procesamiento del lenguaje natural (NLP) están proporcionando conocimientos y predicciones vitales sobre la propagación del virus y la eficacia y el impacto de las contramedidas. Es posible que los modelos anteriores a COVID basados en datos históricos ya no sean válidos. Las técnicas de IA como el aprendizaje por refuerzo y el aprendizaje distribuido están creando sistemas más adaptables y flexibles para manejar situaciones comerciales complejas, por ejemplo, los sistemas basados en agentes pueden modelar y estimular sistemas complejos, particularmente ahora cuando los modelos anteriores a COVID basados en datos históricos, pueden dejar de ser válidos (Gartner, 2020). A continuación, se describirán las tecnologías y los sectores que han logrado un mayor desarrollo durante la pandemia y con crecimiento prometedor para los próximos años.
La Cuarta Revolución Industrial ha representado un cambio fundamental en la forma en que vivimos, trabajamos y nos relacionamos unos con otros. Es un nuevo capítulo en el desarrollo humano, posibilitado por extraordinarios avances tecnológicos acordes con los de la primera, segunda y tercera revoluciones industriales (World Economic Forum, 2021). Estos avances están fusionando los mundos físico, digital y biológico de formas que crean grandes promesas y peligros potenciales. La velocidad, amplitud y profundidad de esta revolución nos está obligando a repensar cómo se desarrollan los países, cómo las organizaciones crean valor e incluso qué significa ser humano. La Cuarta Revolución Industrial es más que un cambio impulsado por la tecnología; es una oportunidad para ayudar a todos, incluidos los líderes, los responsables políticos y las personas de todos los grupos de ingresos y naciones a aprovechar las tecnologías convergentes para crear un futuro inclusivo y centrado en el ser humano. La verdadera oportunidad es mirar más allá de la tecnología y encontrar formas de brindar al mayor número de personas la capacidad de impactar positivamente en sus familias, organizaciones y comunidades.

Por lo expuesto, las empresas deben adaptarse rápidamente a esta realidad. La clave para seguir siendo competitivo es garantizar que se vuelva a capacitar la fuerza laboral existente y que las generaciones futuras estén equipadas con las habilidades adecuadas para tener éxito.

\section{Perfiles ocupacionales dinamizados a nivel mundial en el último año}

En las condiciones adecuadas, la adopción de la tecnología puede fomentar el Desarrollo Industrial Inclusivo y Sostenible (ISID, por sus siglas en inglés) y el logro de los Objetivos de Desarrollo Sostenible (ODS). La innovación tecnológica impulsa el ISID a través de nuevos productos y nuevos procesos. Las nuevas tecnologías son la base del éxito del ISID 
al permitir la creación de nuevos bienes y servicios, lo que conduce a su vez a la aparición de nuevas industrias y también apoya un aumento en la eficiencia de la producción, bajando los costos, los precios y abriendo el consumo al mercado de masas, o aumenta las ganancias, con posibles efectos de seguimiento para la inversión. En el contexto adecuado, las nuevas tecnologías también pueden promover la sostenibilidad ambiental y la inclusión social (United Nations Industrial Development Organization, 2019).

La implementación las nuevas tecnologías en la industria puede conducir a innovaciones de productos, lo que resulta en la aparición de nuevas industrias y generación de empleos de valor agregado. Esto apoya la industrialización y la inclusión social. Cuando estas innovaciones están orientadas a reducir los impactos ambientales, al introducir la fabricación ecológica, también promueven la sostenibilidad ambiental del proceso industrial.

Un estudio llevado a cabo por ManPower Group, reveló que la demanda de profesionales relacionados con la tecnología de la información (TI) y de la manufactura será una de las tendencias que lleva al aumento de personal. Justamente el estudio señala que, para el caso de la TI, un $6 \%$ de las companías planean aumentar el personal que cuente con las competencias correspondientes, destacándose el pensamiento crítico, una alta capacidad de aprendizaje y la programación. En lo que corresponde a la manufactura, $39 \%$ de los líderes empresariales anticipa que requerirá a personal de esta área a corto plazo, que tenga características como gestión de máquinas, alta capacidad de aprendizaje y destrezas para resolver problemas complejos (Manpower Group, 2019a). El crecimiento también vendrá en otras áreas como las de servicio al cliente e ingeniería y gerencias; en el caso de los cargos administrativos, las vacantes se reducirán, aunque la empleabilidad en general no presentará grandes cambios. No obstante, uno de los puntos destacados del estudio es la escasa disponibilidad de ese "talento tecnológico" al que las empresas pretenden apostar, sumado a que los requisitos exigidos por los empleadores en materia de educación y experiencia laboral son desequilibrados frente a lo que existe actualmente.

Cada uno de los factores que se identificaron anteriormente tiene implicaciones para la cantidad, naturaleza, condiciones y transiciones del mercado laboral en general. Aún no está claro hasta qué punto la demanda de mano de obra está cambiando en respuesta a la adopción de tecnología que se ha producido desde el inicio de COVID-19, muchos destacan los posibles impactos negativos de los trabajadores en ciertos sectores u ocupaciones de la "automatización forzada" (Institute for the future of work, 2021). Esto es especialmente preocupante dado que los países están pasando en la actualidad de un período prolongado de aumento del empleo a uno de rápido aumento del desempleo. Hasta ahora, esto ha estado exacerbando las desigualdades preexistentes y creando riesgos reales de cicatrización en el mercado laboral, en particular para ciertos grupos demográficos, como los trabajadores más jóvenes y las mujeres, fenómeno que se ha demostrado en estudios desarrollados en Latinoamérica.

La pérdida de empleo debido a la recesión pandémica obligó a muchos trabajadores a explorar opciones de trabajo por cuenta propia. La experiencia de trabajar de forma remota también les dio a muchos trabajadores la confianza para finalmente ir por su cuenta. En 2020, se estimaba que 59 millones de estadounidenses laboraban como trabajadores independientes, el $36 \%$ de la fuerza laboral total de EE. UU. (Upwork, 2020), este factor se presenta más elevado en las estadísticas de las fuentes de Latinoamérica, por ejemplo, el Banco Central de la República Dominicana relaciona que se mantiene desde hace tres años una tasa de informalidad del $56 \%$, con actualización al tercer trimestre de 2020 (Banco Central, 2021).

Por ello, la evidencia sugiere que en 2021 se necesitará una consideración rápida de políticas impul- 
sadas destinadas a mejorar la adopción responsable de tecnología en la "larga fila" de las PYMEs. Dado el aumento del desempleo y el desplazamiento del mercado laboral debido a la pandemia, también está claro que los programas para fomentar la adopción de tecnología deben complementarse con programas audaces para apoyar la transición de los trabajadores, incluida la mejora y la recapacitación, destinados a construir un futuro más inteligente, sostenible e inclusivo.

Para aquellos de nosotros que tuvimos la suerte de poder hacerlo, trabajar desde casa fue la experiencia definitoria de 2020, pero el privilegio no estaba abierto a todos. Los trabajos que se pueden realizar de forma remota suelen estar ocupados por personas con mayor nivel educativo y mejor remuneración, según un estudio respaldado por la UE (Milasi et al., 2020).

La pandemia afectó más duro a aquellos que no podían trabajar remotamente. Los trabajadores no remotos en los EE. UU. tenían más de tres veces mayor probabilidad de perder sus trabajos durante la pandemia que sus pares que trabajaban a distancia, según un documento publicado por la Oficina Nacional de Investigación Económica en agosto (Angelucci et al., 2020). Esta discrepancia fue aún mayor entre las mujeres, los afroamericanos, los hispanos y las personas sin títulos universitarios, encontró el estudio. Los trabajadores que no vivían en lugares remotos también tenían una salud respiratoria más deficiente que sus colegas confinados en casa.

Los empleadores ahora están rediseñando su modelo de administración del talento humano con la "nueva normalidad" para sus trabajadores. Empresas como Google, Microsoft y American Express han anunciado planes para convertir el trabajo remoto en un elemento permanente; un estudio del Instituto de Directores del Reino Unido en octubre encontró que el $74 \%$ de los empleadores planea hacer lo mismo (IoD, 2020), esto se corrobora en otras partes del mundo, por ejemplo, casi tres de cada cuatro directores ejecutivos planean trasladar al menos el cinco por ciento de los empleados, que anteriormente estaban en el sitio, a puestos permanentemente remotos después de COVID-19 (McKinsey, 2020).

Los sectores de la Salud y de Tecnología de la Información son proyectados como aquellos con mayor posibilidad de generar mayor número de empleos en el futuro en EE. UU. Según datos recientes del Departamento de Trabajo de Estados Unidos, el envejecimiento de la población incrementará la demanda de trabajadores de la salud como médicos, enfermeras, fisioterapeutas, asistentes de salud en el hogar y farmacéuticos. Esta tendencia se ha corroborado con los resultados de la actual pandemia, pues se han incrementado las solicitudes de trabajadores de la salud en el último año.

En la última década, el incremento de la demanda de ayudantes de salud en el hogar y las asistentes de cuidado personal ha llegado al $70 \%$, adicionalmente, las enfermeras registradas se han incrementado en un $26 \%$ (Forbes, 2020). Trabajos emergentes, no tradicionales, tales como el Técnico en Salud Conductual, están experimentando un aumento anual del $32 \%$. La mayor cobertura de seguro de salud para el tratamiento de salud mental y abuso de sustancias ha creado esta mayor demanda de Técnicos de Salud del Comportamiento, muchos de los cuales se especializan en pacientes que tienen autismo o trastornos del comportamiento y están trabajando con niños en entornos escolares (LinkedIn, 2020).

De acuerdo con la última data disponible de LinkedIn, durante el año 2020 se contrataron un total de 145.270 enfermeros y enfermeras en EE. UU., cifra que ubica a este perfil ocupacional con mayor demanda. De igual manera, a enero de 2021, se requerían 48.247 personas con licencia en esta labor. Igualmente, el personal de apoyo sanitario, que se puede capacitar de forma remota y no requiere un título de cuatro 
años, ha sido altamente demandado por el mercado de la salud en ese país, llegando a 44.494 puestos, existiendo vacantes de 81.735 empleos. También las cifras de vacantes de especialista en salud mental son elevadas, dado que se necesitan 16.974, presentando un $24 \%$ de incremento en contrataciones frente al 2019 (LinkedIn, 2021).

Por otra parte, en la medida que se desarrolle más tecnología, los profesionales de TI, como programadores, especialistas en seguridad y administradores, seguirán teniendo una gran demanda (Salary.com, 2020).

\section{Competencias laborales emergentes}

Desde la arista de la demanda del trabajo, durante el último año, las competencias laborales que solicitaron las empresas a nivel mundial se centraron en pensamiento crítico, actitud y capacidad de aprendizaje continuos y, debido a las consecuencias de la pandemia, se han requerido en mayor proporción habilidades y valores tales como resiliencia, adaptabilidad al cambio y alto nivel de responsabilidad (Institute for the future of work, 2021; Forbes, 2021), esto se explica porque una buena parte de las empresas adoptaron nuevas tecnologías digitales (por ejemplo, tecnologías de trabajo remoto o computación en la nube), así como mejores prácticas desde el inicio de la pandemia, e invirtieron en nuevas capacidades digitales (Riom y Valero, 2020). Guardando congruencia con lo anterior, en el futuro próximo, participar activamente en los segmentos de mercado de la Cuarta Revolución Industrial requerirá de habilidades interactivas y creativas. El mayor uso de las tecnologías digitales y el aumento de las tareas intensivas en conocimiento también han provocado cambios importantes en los requisitos de habilidades de los empleados, fenómeno que continuará en aumento, debido a que se ha evidenciado la importancia del trabajo remoto por el confinamiento obligado.
Desde la arista de la oferta del trabajo, un estudio desarrollado recientemente por Korn Ferry (2020b), evidenció que, para 2030, se prevé que la escasez mundial de talento alcance los 85,2 millones de trabajadores. Las empresas de todo el mundo corren el riesgo de perder USD 8,4 billones en ingresos debido a la falta de talento calificado. Las tendencias mundiales del mercado y la tecnología son los principales factores que afectan la creciente escasez de talento mundial. Este fenómeno ha sido corroborado en otra investigación, donde se demostró que el $87 \%$ de las organizaciones ya están experimentando una escasez de talento o esperan enfrentarla en unos pocos ańos (McKinsey, 2020). Por ejemplo, la escasez de desarrolladores de software en los países nórdicos ya es un problema: solo Suecia carecerá de 70.000 especialistas TI o competencias relacionadas con la tecnología digital para 2022 (Daxx, 2020a). Mientras tanto, Israel está experimentando una escasez de desarrolladores de software del $15 \%$ (Daxx, 2020b), al mismo tiempo que se encuentra entre las tres naciones emergentes de tecnología de más rápido desarrollo. Por esto, las industrias de análisis de datos, TI, dispositivos móviles y/o diseño web son las más propensas a esperar interrupciones debido a la brecha de habilidades y la escasez de talento de TI.

En los países latinoamericanos consultados ocurre el mismo fenómeno, siendo Costa Rica y Panamá aquellos con menos problemática para la consecución de talento (35\%), y México y Argentina aquellos con mayores dificultades (50\% y $54 \%$, respectivamente). Los oficios calificados son los que más dificultad presentan (Manpower Group, 2019b). En el contexto dominicano, el informe "Estudio de detección de necesidades de capacitación y demanda laboral 2019" mostró que en los territorios de alto nivel de pobreza en la República Dominicana existe una importante demanda de capacitación, ya que el $84.5 \%$ de los empleadores indicaron que los trabajadores requieren formación o capacitación específica, el $40 \%$ reportó algún 
nivel de dificultad para conseguir los perfiles requeridos y el $78 \%$ de los consultados entiende que los prospectos disponibles necesitan más formación (Vicepresidencia de la República Dominicana, 2019). El reporte añade que existen unos $738 \mathrm{mil}$ potenciales demandantes de servicios de formación técnico profesional y los empleadores requieren que sus colaboradores cuenten con múltiples habilidades o competencias, demandando capacitación técnica, una buena educación general, dominio de ofimática, idiomas y habilidades para el trabajo colaborativo.

Así las cosas, participar activamente en la Cuarta Revolución Industrial (CRI) requerirá de habilidades interactivas y creativas. El mayor uso de las tecnologías digitales y el aumento de las tareas intensivas en conocimiento también han provocado cambios importantes en los requisitos de habilidades de los empleados, fenómeno que continuará en aumento como se ha evidenciado con el trabajo remoto por el confinamiento obligado. Las competencias sociales, de comunicación y creativas han ganado sustancial importancia, igual que los requerimientos de habilidades complejas de resolución de problemas y el pensamiento creativo. En general, cada vez más empleados dependen de dispositivos y tecnologías digitales, realizan trabajos de conocimiento y tareas no rutinarias, y están supeditados a la interacción social, la colaboración y la creatividad para realizar su trabajo. Es probable que estas tendencias se vuelvan aún más marcadas en los próximos años como respuesta al crecimiento exponencial de la CRI (Deloitte, 2019a).

$\mathrm{Al}$ respecto, si se tiene en cuenta la formación requerida de la población, la revisión bibliográfica de los países de América y Europa coincide en que el desafío será abordar los desajustes locales (regiones de cada país) y ayudar a los trabajadores a adquirir nuevas habilidades para el trabajo del futuro. Este fenómeno se debe a que los trabajadores con nivel educativo de secundaria o menor, en Estados Unidos, tienen cuatro veces más probabilidades de ser reem- plazados por la automatización (McKinsey, 2019), y en la Unión Europea más del 70 \% de las empresas han manifestado que la falta de personal con las habilidades digitales adecuadas es un obstáculo para la inversión, por lo que encontrar y mantener un trabajo en la nueva economía podría ser una tarea imposible para ellos (European Commission, 2021). No obstante, la CRI está facilitando el acceso a la formación inclusiva, por medio del microaprendizaje, simulaciones, gamificación (videojuegos divertidos con retroalimentación inmediata, autonomía de decisión, situaciones abiertas, reintentos infinitos, progresividad, con reglas claras y sencillas) y entrenamiento virtual con plataformas interactivas. Se trata de nuevas metodologías que resultan estimulantes para el adquirente del conocimiento, tienen precios que por lo general representan una pequeña fracción del coste de la formación tradicional y, en muchos casos, ofrecen calidades de primer nivel.

\section{Metodología}

La información utilizada para definir las características de la demanda de trabajo se obtuvo a partir del análisis de la demanda de personal diaria solicitada por las cinco bolsas o agencias de empleo con mayor número de avisos web de requerimientos de empleados en la República Dominicana. La consecución de la data se realizó en dos momentos, antes de la pandemia y un año después. La primera medición correspondió a la data recolectada en los meses de diciembre del año 2019 y enero del año 2020 (en adelante período dic19-ene20), la segunda medición tomó la data de los meses de diciembre de 2020 y enero de 2021 (en adelante dic20-ene21). Esta técnica permite conocer el perfil de los cargos solicitados por las empresas demandantes, la cual ha sido validada previamente en otros estudios (Cárdenas y Guataquí, 2015; Barna Management School y COPARDOM, 2020).

Las variables seleccionadas dependieron de la disponibilidad de información en las plataformas, dentro de las cuales se lograron determinar las demográficas 
como sexo, edad mínima y máxima exigidas, así como variables típicas de la descripción de los cargos: formación y experiencia exigidas, perfil ocupacional descrito en la vacante y competencias, tanto blandas (habilidades), como duras (dominio de TICs).

\section{Resultados}

En total se recolectaron 2008 registros en el período dic19-ene20 y 2009 registros del período dic20-ene21. La distribución de los datos por provincia se observa en la tabla 1 , de donde se deduce que en Santo Domingo se encontró la mayor parte de la demanda laboral del país, incrementándose su participación del $71 \%$ al $80 \%$ en el segundo período de análisis, seguido por Santiago (11\% y $10 \%$, respectivamente).

Tabla 1. Número de registros procesados por provincia por periodo

\begin{tabular}{|l|c|c|c|c|}
\hline \multicolumn{1}{|c|}{ Período } & \multicolumn{2}{|c|}{ dic19-ene20 } & \multicolumn{2}{c|}{ dic20-ene21 } \\
\hline Azua & $\mathrm{n}$ & $\%$ & $\mathrm{n}$ & $\%$ \\
\hline Baní & 5 & $0,2 \%$ & 0 & $0,0 \%$ \\
\hline Bávaro & 4 & $0,2 \%$ & 20 & $1,0 \%$ \\
\hline Bellavista & 78 & $3,9 \%$ & 47 & $2,3 \%$ \\
\hline Boca chica & 1 & $0,0 \%$ & 0 & $0,0 \%$ \\
\hline Bonao & 10 & $0,5 \%$ & 0 & $0,0 \%$ \\
\hline Haina & 8 & $0,4 \%$ & 0 & $0,0 \%$ \\
\hline Higüey & 40 & $2,0 \%$ & 0 & $0,0 \%$ \\
\hline La Romana & 42 & $2,1 \%$ & 12 & $0,6 \%$ \\
\hline La Vega & 63 & $3,1 \%$ & 21 & $1,0 \%$ \\
\hline Promoción y ventas & 0 & $0,0 \%$ & 15 & $0,7 \%$ \\
\hline Puerto Plata & 1 & $0,0 \%$ & 0 & $0,0 \%$ \\
\hline Punta Cana & 40 & $2,0 \%$ & 14 & $0,7 \%$ \\
\hline Sabana Palenque & 4 & $0,2 \%$ & 0 & $0,0 \%$ \\
\hline San Cristóbal & 22 & $1,1 \%$ & 39 & $1,9 \%$ \\
\hline San Pedro de Macorís & 26 & $1,3 \%$ & 33 & $1,6 \%$ \\
\hline Santiago & 226 & $11,3 \%$ & 192 & $9,6 \%$ \\
\hline Santo Domingo & 1434 & $71,4 \%$ & 1604 & $79,8 \%$ \\
\hline Total base & 2008 & $100,0 \%$ & 2009 & $100,0 \%$ \\
\hline
\end{tabular}

A continuación, se exponen los diferentes hallazgos en cada factor componente.

\section{Sexo}

$\mathrm{Al}$ tener en cuenta si las empresas detallan el sexo en sus solicitudes, la tabla 2 muestra que el $32 \%$ de las empresas describen el sexo en el perfil, existiendo en el segundo período (dic21-ene21) una mayor solicitud de hombres (63\%).

Tabla 2. Solicitud por sexo, por periodo

\begin{tabular}{|c|c|c|c|c|}
\hline Período & \multicolumn{2}{|c|}{ dic19-ene20 } & \multicolumn{2}{c|}{ dic20-ene21 } \\
\hline Sexo & $\mathrm{n}$ & $\%$ & $\mathrm{n}$ & $\%$ \\
\hline Hombre & 352 & $60,6 \%$ & 407 & $63,1 \%$ \\
\hline Mujer & 229 & $39,4 \%$ & 238 & $36,9 \%$ \\
\hline Especifica & 581 & $28,9 \%$ & 645 & $32,1 \%$ \\
\hline Sin especificar & 1428 & $71,1 \%$ & 1365 & $67,9 \%$ \\
\hline Total & 2009 & $100,0 \%$ & 2010 & $100,0 \%$ \\
\hline
\end{tabular}

\section{Edad y años de experiencia exigidos, según cargo solicitado}

El promedio de la edad mínima exigida es de 24 años (tabla 3), aspecto que no cambió en el último período, en tanto, la edad máxima se incrementó en un año (diferencias significativas ${ }^{1}$ ), ascendiendo a 40.6 años, sugiriendo una leve mejora en la inclusión de población mayor.

Tabla 3. Edad minima y máxima reportada por la demanda de trabajo (total pais)

\begin{tabular}{|l|c|c|}
\hline & \multicolumn{2}{|c|}{ Período } \\
\hline \multicolumn{1}{|c|}{ Media } & dic19-ene20 & dic20-ene21 \\
\hline Edad min & 23,7 & 23,7 \\
\hline Edad máx & 39,5 & 40,6 \\
\hline
\end{tabular}

La mayoría de las empresas (72 \%) solicita experiencia para ocupar los cargos vacantes. De ellas, el $42 \%$ exigen que sea mayor a un ańo y otro $42 \%$ mayor a dos ańos, indicando la baja intención de contratar a nuevos empleados sin experiencia (tabla 4). Estas cifras no cambiaron desde la medición del período anterior.

${ }^{1}$ Prueba U de Mann-Whitney para muestras independientes 
Tabla 4. Experiencia reportada por la demanda de trabajo, por periodo (total pais)

\begin{tabular}{|l|c|c|c|c|}
\hline \multicolumn{1}{|r|}{ Período } & \multicolumn{2}{|c|}{ dic19-ene20 } & \multicolumn{2}{c|}{ dic20-ene21 } \\
\hline Experiencia & $\mathrm{n}$ & $\%$ & $\mathrm{n}$ & $\%$ \\
\hline Mayor a un año & 605 & $41,9 \%$ & 644 & $42,1 \%$ \\
\hline Mayor a dos años & 607 & $42,0 \%$ & 635 & $41,5 \%$ \\
\hline Mayor a 5 años & 167 & $11,6 \%$ & 168 & $11,0 \%$ \\
\hline Mayor a 3 años & 50 & $3,5 \%$ & 69 & $4,5 \%$ \\
\hline Mayor a 10 años & 0 & $0,0 \%$ & 5 & $0,3 \%$ \\
\hline Mayor a 4 años & 10 & $0,7 \%$ & 5 & $0,3 \%$ \\
\hline Mayor a 6 años & 2 & $0,1 \%$ & 1 & $0,1 \%$ \\
\hline Sin experiencia & 4 & $0,3 \%$ & 4 & $0,3 \%$ \\
\hline No especifica & 561 & $38,8 \%$ & 477 & $31,2 \%$ \\
\hline Total & 1445 & $100,0 \%$ & 1531 & $100,0 \%$ \\
\hline
\end{tabular}

\section{Formación}

En el año 2014, el "Estudio prospectivo sobre demandas de cualificaciones técnico- profesionales en República Dominicana", encontró que un poco más del $60 \%$ de los empleadores formales solicitaron que los candidatos a ocupar los puestos de trabajo en los niveles de base y medio de la pirámide ocupacional, tuvieran como nivel mínimo la educación secundaria completa, corroborando que la demanda exige formación de, al menos, secundaria. Seis años después, según la información recolectada en el estudio desarrollado en 2020 (véase tabla 5, período dic19-ene20), la tendencia parece haber evolucionado en función de un mayor nivel de exigencia, pues el $17 \%$ de la demanda de trabajadores solicita personal de formación secundaria, pero el restante $(83 \%)$ requieren personas con capacitación superior, esto es, estudiantes universitarios (27\%), técnicos (6\%), profesionales (44\%) y con estudios de posgrado (10\%). Al actualizar esta data con la medición del segundo período (dic20-ene21), el mercado se mantuvo con su nivel de exigencia en formación.

Tabla 5. Formación exigida, por periodo

\begin{tabular}{|l|c|c|c|c|}
\hline \multicolumn{1}{|c|}{ Período } & \multicolumn{2}{|c|}{ dic19-ene20 } & \multicolumn{2}{c|}{ dic20-ene21 } \\
\hline Formación exigida & $\mathrm{n}$ & $\%$ & $\mathrm{n}$ & $\%$ \\
\hline profesional nivel medio & 875 & $43,8 \%$ & 948 & $47,2 \%$ \\
\hline estudiante universitario & 541 & $27,1 \%$ & 529 & $26,4 \%$ \\
\hline bachiller & 348 & $17,4 \%$ & 292 & $14,5 \%$ \\
\hline máster & 209 & $10,5 \%$ & 136 & $6,8 \%$ \\
\hline técnico & 114 & $5,7 \%$ & 106 & $5,3 \%$ \\
\hline Total & 1996 & $100,0 \%$ & 2007 & $100,0 \%$ \\
\hline
\end{tabular}

Este proceder de las empresas ha exacerbado el problema de empleabilidad de la mujer, explicando la mayor tasa de desempleo del colectivo femenino (gráfico 1), comportamiento que se ha acentuado en los últimos trimestres de medición, donde se observa una mayor distancia en la empleabilidad entre hombres y mujeres.

\section{Gráfico 1. Nivel de empleabilidad por sexo}

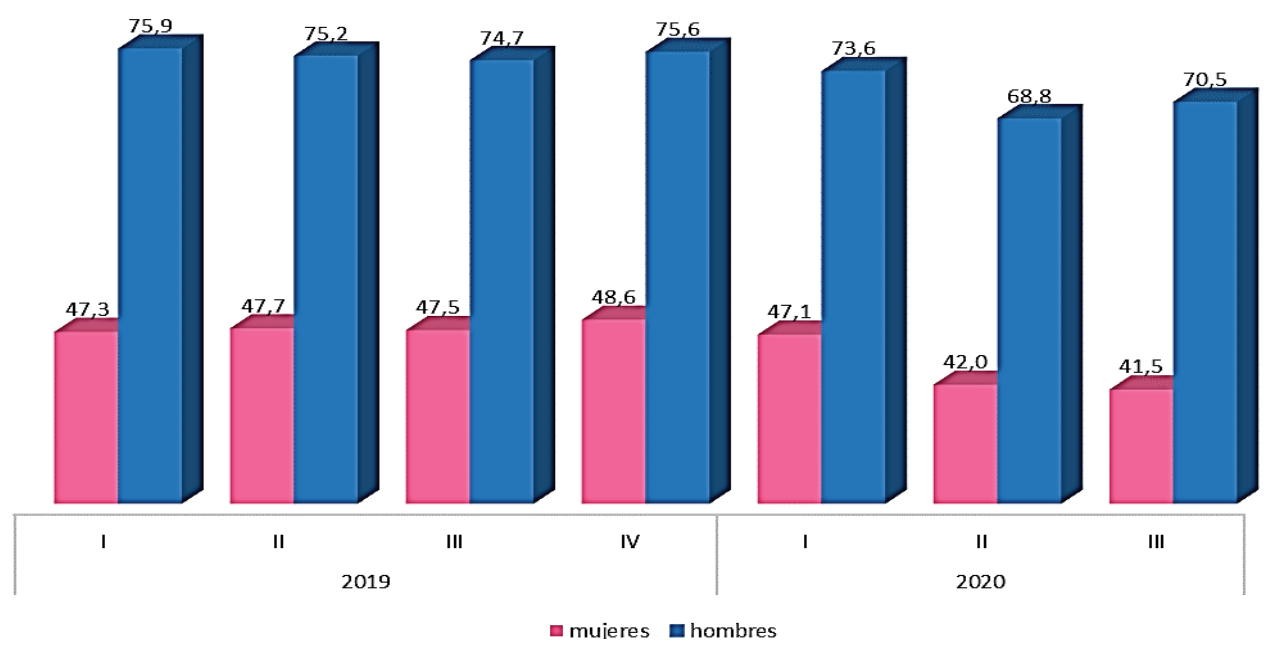

Nota: elaboración propia con data del ENCFT. 


\section{Experiencia exigida}

La tabla 6 describe la experiencia requerida por las empresas en los períodos dic19-ene20 y dic20-ene21. Se observa que la atención al cliente es la principal experiencia solicitada desde el ańo pasado. El mayor cambio se presentó en análisis de información, seguido por planeación, experiencias que hacen parte de la CRI, según lo que se ha manifestado en los capítulos precedentes.

Tabla 6. Competencias exigidas por las empresas

\begin{tabular}{|c|c|c|c|c|}
\hline Período & \multicolumn{2}{|c|}{ dic19-ene20 } & \multicolumn{2}{|c|}{ dic20-ene21 } \\
\hline Experiencia exigida & $\mathrm{n}$ & $\%$ & $\mathrm{n}$ & $\%$ \\
\hline Atención al cliente & 742 & $37,6 \%$ & 835 & $44,8 \%$ \\
\hline Administrativo & 272 & $13,8 \%$ & 401 & $21,5 \%$ \\
\hline Análisis de info & 193 & $9,8 \%$ & 389 & $20,9 \%$ \\
\hline Supervisión /manejo de personal & 308 & $15,6 \%$ & 374 & $20,1 \%$ \\
\hline Ventas externas & 345 & $17,5 \%$ & 341 & $18,3 \%$ \\
\hline Planeación & 129 & $6,5 \%$ & 307 & $16,5 \%$ \\
\hline Supervisión de procesos & 124 & $6,3 \%$ & 287 & $15,4 \%$ \\
\hline Contabilidad & 297 & $15,1 \%$ & 253 & $13,6 \%$ \\
\hline Manejo conflictos & 129 & $6,5 \%$ & 186 & $10,0 \%$ \\
\hline Mercadeo & 187 & $9,5 \%$ & 181 & $9,7 \%$ \\
\hline Ing. industrial & 88 & $4,5 \%$ & 163 & $8,7 \%$ \\
\hline Logística & 73 & $3,7 \%$ & 147 & $7,9 \%$ \\
\hline Ingeniería de sistemas & 37 & $1,9 \%$ & 146 & $7,8 \%$ \\
\hline Inventario & 110 & $5,6 \%$ & 130 & $7,0 \%$ \\
\hline Búsqueda de info & 87 & $4,4 \%$ & 108 & $5,8 \%$ \\
\hline Publicidad, RRPP & 48 & $2,4 \%$ & 94 & $5,0 \%$ \\
\hline Transporte & 69 & $3,5 \%$ & 81 & $4,3 \%$ \\
\hline Desarrollador/programador & 85 & $4,3 \%$ & 80 & $4,3 \%$ \\
\hline Enfermería/medicina/salud & 61 & $3,1 \%$ & 77 & $4,1 \%$ \\
\hline Hotelería y turismo & 31 & $1,6 \%$ & 71 & $3,8 \%$ \\
\hline Soporte técnico & 56 & $2,8 \%$ & 70 & $3,8 \%$ \\
\hline Mantenimiento equipos & 54 & $2,7 \%$ & 69 & $3,7 \%$ \\
\hline Diseño gráfico & 34 & $1,7 \%$ & 67 & $3,6 \%$ \\
\hline $\mathrm{RRHH}$ & 86 & $4,4 \%$ & 65 & $3,5 \%$ \\
\hline Bodegas & 34 & $1,7 \%$ & 55 & $3,0 \%$ \\
\hline Gerencia & 48 & $2,4 \%$ & 47 & $2,5 \%$ \\
\hline Psicología & 15 & $0,8 \%$ & 44 & $2,4 \%$ \\
\hline Ing. eléctrica & 9 & $0,5 \%$ & 44 & $2,4 \%$ \\
\hline Productos financieros & 77 & $3,9 \%$ & 40 & $2,1 \%$ \\
\hline Servicios / oficios varios & 47 & $2,4 \%$ & 39 & $2,1 \%$ \\
\hline Mecánica industrial & 7 & $0,4 \%$ & 39 & $2,1 \%$ \\
\hline Capacitación/formación & 48 & $2,4 \%$ & 36 & $1,9 \%$ \\
\hline Cajero & 47 & $2,4 \%$ & 32 & $1,7 \%$ \\
\hline Auditoría & 58 & $2,9 \%$ & 30 & $1,6 \%$ \\
\hline Menos de 30 & 631 & $32,0 \%$ & 500 & $26,8 \%$ \\
\hline Total menciones & 1971 & $98,1 \%$ & 1864 & $92,7 \%$ \\
\hline Total respondientes & 2009 & $101,9 \%$ & 2010 & $107,8 \%$ \\
\hline
\end{tabular}

\section{Perfil ocupacional}

El área ocupacional con mayor demanda de empleados es: técnicos y profesionales del nivel medio (véase tabla 7).

Tabla 7. Perfil ocupacional de los puestos de trabajo solicitados

\begin{tabular}{|l|c|c|c|c|}
\hline Periodo & \multicolumn{2}{|c|}{ dic19-ene20 } & \multicolumn{2}{c|}{ dic20-ene21 } \\
\hline Perfil ocupacional & $\mathrm{n}$ & $\%$ & $\mathrm{n}$ & $\%$ \\
\hline $\begin{array}{l}\text { Técnicos y profesionales } \\
\text { del nivel medio }\end{array}$ & 383 & $19,1 \%$ & 1535 & $76,4 \%$ \\
\hline $\begin{array}{l}\text { Trabajadores de los } \\
\text { servicios y vendedores de }\end{array}$ & 483 & $24,1 \%$ & 172 & $8,6 \%$ \\
\hline Directores y gerentes & 157 & $7,8 \%$ & 125 & $6,2 \%$ \\
\hline $\begin{array}{l}\text { Oficiales, operarios y } \\
\text { artesanos de artes }\end{array}$ & 217 & $10,8 \%$ & 73 & $3,6 \%$ \\
\hline $\begin{array}{l}\text { Profesionales científicos e } \\
\text { intelectuales }\end{array}$ & 110 & $5,5 \%$ & 59 & $2,9 \%$ \\
\hline $\begin{array}{l}\text { Personal de apoyo } \\
\text { administrativo }\end{array}$ & 584 & $29,2 \%$ & 40 & $2,0 \%$ \\
\hline Ocupaciones militares & 0 & $0,0 \%$ & 2 & $0,1 \%$ \\
\hline Ocupaciones elementales & 66 & $3,3 \%$ & 2 & $0,1 \%$ \\
\hline $\begin{array}{l}\text { Operadores de } \\
\text { instalaciones y máquinas }\end{array}$ & 2 & $0,1 \%$ & 1 & $0,0 \%$ \\
\hline $\begin{array}{l}\text { Agricultores y trabajadores } \\
\text { calificados agropecuarios, }\end{array}$ & 0 & $0,0 \%$ & 1 & $0,0 \%$ \\
\hline Total & 2002 & $100,0 \%$ & 2010 & $100,0 \%$ \\
\hline
\end{tabular}

Esta área ocupó el tercer lugar en el año anterior, de manera que sustituyó la demanda de personal de apoyo administrativo, así como a los trabajadores de los servicios y vendedores de comercios, sectores que fueron los más golpeados por la pandemia (Banco Central, 2020).

\section{Competencias solicitadas según el cargo}

En el año 2014, las competencias transversales de mayor relevancia para los empleadores fueron el trabajo en equipo ( $48 \%$ ) y las herramientas de comunicación oral y escrita (43\%). En 2020, las competencias solicitadas con mayor frecuencia fueron el trabajo en equipo (31\%), el enfoque a resultados (31\%), la destreza para comunicar $(27 \%)$ y la 
responsabilidad (27\%), indicando que los niveles de exigencia en competencias se han ido incrementando con el paso del tiempo (tabla 8). En el 2021 cambiaron de manera significativa las proporciones de competencias solicitadas por las empresas, puesto que se duplicó la cantidad de empresas exigiendo el enfoque en resultados (66 \%) y destreza para comunicar (41\%).

Tabla 8. Competencias exigidas por las empresas

\begin{tabular}{|l|c|c|c|c|}
\hline \multicolumn{1}{|c|}{ Período } & \multicolumn{2}{|c|}{ dic19-ene20 } & \multicolumn{2}{c|}{ dic20-ene21 } \\
\hline Competencias & $\mathrm{n}$ & $\%$ & $\mathrm{n}$ & $\%$ \\
\hline Enfoque en resultados & 564 & $28,1 \%$ & 1324 & $65,9 \%$ \\
\hline Destreza para comunicar & 494 & $24,6 \%$ & 823 & $40,9 \%$ \\
\hline Trabajo en equipo & 634 & $31,6 \%$ & 788 & $39,2 \%$ \\
\hline Responsabilidad & 558 & $27,8 \%$ & 750 & $37,3 \%$ \\
\hline Dinamismo/Proactividad & 357 & $17,8 \%$ & 442 & $22,0 \%$ \\
\hline Liderazgo & 127 & $6,3 \%$ & 426 & $21,2 \%$ \\
\hline Empatía & 266 & $13,2 \%$ & 400 & $19,9 \%$ \\
\hline Integridad & 240 & $11,9 \%$ & 279 & $13,9 \%$ \\
\hline Iniciativa & 171 & $8,5 \%$ & 155 & $7,7 \%$ \\
\hline Creatividad & 76 & $3,8 \%$ & 111 & $5,5 \%$ \\
\hline Flexibilidad & 105 & $5,2 \%$ & 89 & $4,4 \%$ \\
\hline Tolerancia a la frustración & 79 & $3,9 \%$ & 75 & $3,7 \%$ \\
\hline Autoconfianza & 25 & $1,2 \%$ & 38 & $1,9 \%$ \\
\hline Sin especificar & 492 & $24,5 \%$ & 18 & $0,9 \%$ \\
\hline Total menciones & 3696 & $184,0 \%$ & 5700 & $283,6 \%$ \\
\hline Total respondientes & 2009 & $100,0 \%$ & 2010 & $100,0 \%$ \\
\hline
\end{tabular}

Por otra parte, competencias que habían presentado menos menciones en el primer período (dic19-ene20), en la presente medición alcanzaron mayor importancia, tales como liderazgo y responsabilidad. Esta última sugiere que se ha requerido como parte del trabajo remoto. Es importante mencionar que hace un año las empresas solicitaban 1.8 competencias, en tanto, ahora piden 2.8 competencias en promedio, indicando un mayor nivel de exigencia.

Estas destrezas han sido mencionadas en múltiples estudios relacionados con el futuro del trabajo, donde se demuestra que, además de las habilidades duras (dominio del inglés, titulación a nivel superior, experiencia en TICs y plataformas digitales), las empresas que se encuentran compitiendo en mercados y segmentos asociados a la CRI, exigen las habilidades blandas, tales como pensamiento crítico, creatividad, solución de problemas complejos, interacción social, trabajo en equipo, destrezas en comunicación, alta intención y capacidad de aprendizaje, inteligencia cultural, resiliencia, adaptabilidad al cambio y responsabilidad (Forbes, 2021; Institute for the future of work, 2021; Korn Ferry, 2020a).

\section{Formación en TICs}

En el período de dic-19-ene20, el 56\% delas empresas estuvieron solicitando personal con competencias en TICs, en tanto, en la medición actualizada a enero 2021, se incrementó el valor a $62 \%$ (tabla 9), lo cual corresponde a las tendencias de la Tercera revolución industrial y un requisito imprescindible para participar activamente en los mercados y segmentos de la CRI. De igual manera, este requisito se incrementó probablemente debido a la necesidad de contratar personal con competencias para desarrollar trabajo remoto, aspecto imprescindible para desempeñar muchos perfiles ocupacionales durante la pandemia y puestos del futuro del trabajo (Organización Internacional del Trabajo, 2019; CEPAL, 2020).

Tabla 9. Competencias en TICs solicitadas, por año

\begin{tabular}{|l|r|c|r|c|}
\hline & Periodo & \multicolumn{2}{|c|}{ dic19-ene20 } & \multicolumn{2}{c|}{ dic20-ene21 } \\
\hline \multicolumn{1}{|c|}{ TIC } & \multicolumn{1}{c|}{$\mathrm{n}$} & \multicolumn{1}{c|}{$\%$} & $\mathrm{n}$ & $\%$ \\
\hline Sin especificar & 894 & $44,5 \%$ & 767 & $38,2 \%$ \\
\hline Si exige & 1115 & $55,5 \%$ & 1243 & $61,8 \%$ \\
\hline Total & 2009 & $100,0 \%$ & 2010 & $100,0 \%$ \\
\hline
\end{tabular}

\section{Idiomas}

Al considerar los idiomas como competencias, el $32 \%$ de las empresas exigieron inglés para ocupar los cargos vacantes en el período dic19-ene20, mientras que en la medición de dic-20-ene21 esta proporción se incrementó al $40 \%$, demostrando una mayor exigencia por parte de las empresas solicitantes. 


\section{Conclusiones}

La mayoría de las empresas buscan perfiles de 30 años, como edad mínima, y 45 como máximo, comportamiento que no ha cambiado en el último año. Tan solo una muy pequeña proporción de los empleadores se interesan en personas mayores de 45 años y menores de 25 años. Esta tendencia es similar en otros países latinoamericanos, donde no existe una ley que prohíba a las empresas reclutar personal según la edad o sexo del solicitante, acarreando problemas de desempleo en ciertos grupos poblacionales. No obstante, algunos países optan por estimular la contratación con disminución de impuestos en empresas que vinculen a determinados colectivos. Como ejemplo está Costa Rica, donde se incrementó el desempleo en $120 \%$ en hombres mayores de 45 ańos en el segundo trimestre de 2020, por lo que el presidente decretó una reducción del $5 \%$ del pago de la renta a empresas que tuvieran en su planilla un $10 \%$ de trabajadores mayores de esta edad (La República, 2020).

Una gran parte de las empresas dominicanas solicitan en sus procesos de reclutamiento y selección de personas que los candidatos hayan trabajado en cargos similares durante un año o más, indicando la baja intención de contratar a nuevos empleados sin experiencia alguna, de manera que se excluye a la población joven, intensificando aún más la problemática de desempleo en este grupo etario. En algunos países han mejorado la empleabilidad en colectivos jóvenes por medio de programas tales como ProJovem en Brasil, Jóvenes con Oportunidades en México, Jóvenes con Más y Mejor Trabajo en Argentina, Chile Joven en Chile y Jóvenes a la Obra (antes ProJoven) en Perú y Jóvenes en Acción en Colombia. En México, Brasil, Colombia y Argentina se les otorga a los jóvenes transferencias condicionadas para estimularlos a que concluyan el ciclo de educación superior y tengan mayores opciones de empleo en el sector formal y la posibilidad de acceder a una pensión en el futuro (Misión Colombia envejece, 2020).

En la República Dominicana, la mayor parte de puestos vacantes actuales se concentra en técnicos profesionales de nivel medio, reduciéndose de manera significativa la proporción de solicitudes de personal para labores de administración, ventas y servicios, perfiles solicitados en mayor porcentaje hace un ańo. Este comportamiento sugiere que se redujeron las vacantes disponibles en los empleos asociados a la cadena de valor del sector turístico y de comercio, los económicamente más golpeados por la pandemia (Organización Internacional del Trabajo, 2020).

En los últimos siete años el nivel de educación exigido por las empresas se ha ido incrementando, de tal manera que para el período de análisis de dic20-ene21 se reportó que la mayoría requieren personas con capacitación terciaria $(80 \%)$, evidenciando la gran necesidad de estimular la formación superior y técnica en la población dominicana. Adicionalmente, más de la mitad de los contratantes están solicitando personal con competencias en TICs, tendencia propia de la Tercera revolución industrial y un requisito sine qua non para empezar a aprovechar las oportunidades de la CRI. Por otra parte, las exigencias de conocimientos o dominio del idioma inglés pasaron del $30 \%$ al $40 \%$ en el último año, sugiriendo la necesidad reinante de capacitarse en este idioma. Al respecto, en la web hay disponibilidad de múltiples plataformas interactivas que permiten mejorar las habilidades duras mencionadas anteriormente, $\tan$ solo se requiere la voluntad de las personas, así como el acceso a banda ancha con un equipo adecuado, aspectos que pueden incrementar aún más la brecha social. 
Desde hace siete años las habilidades blandas de mayor relevancia para los empleadores continúan siendo el trabajo en equipo y las herramientas de comunicación, tanto oral como escrita. En la medición del primer período del presente estudio (dic19-ene20) se incluyeron el enfoque a resultados y la responsabilidad. En la medición más reciente (dic20-ene21), se demandaron más personas con competencias en enfoque en resultados, destrezas para comunicar, liderazgo y responsabilidad, evidenciando requerimientos cada vez más exigentes en habilidades blandas. Lo anterior, sumado a que en el período dic20-ene21 las empresas solicitaron un promedio de 2.8 competencias, una más que en el período anterior (dic19-ene20), sugiere que la demanda se está volviendo cada vez más rigurosa, presionando a la oferta del trabajo a formarse en las destrezas y habilidades requeridas.

Por lo anteriormente expuesto, los modelos educativos del país, tanto en el sector público como en el privado, podrían diseñar programas o rediseñar sus currículos, incluyendo competencias duras asociadas a la CRI, tales como las de modelos STEM (acrónimo de los términos en inglés Science, Technology, Engineering and Mathematics (ciencia, tecnología, ingeniería y matemáticas) TICs (Tecnologías de Información y Comunicación) y dominio del idioma inglés, articuladas con habilidades blandas requeridas por la demanda del trabajo, esto es, pensamiento crítico, creatividad, solución de problemas complejos, interacción social, trabajo en equipo, destrezas en comunicación, alta intención y capacidad de aprendizaje, inteligencia cultural, resiliencia, adaptabilidad al cambio y, como valor primordial, alto nivel de responsabilidad.

Futuras investigaciones pueden centrarse en averiguar los perfiles requeridos en cargos de dirección, los cuales corresponden a Head Hunters, quienes probablemente no publican sus necesidades de contratación en la web.

\section{Referencias}

Angelucci, M., Angrisani, M., Bennett, D., Kapteyn, A \&. Schaner, S. (2020). Remote Work and the Heterogeneous Impact of COVID-19 on Employment and Health. National Bureau of Economic Research. (Working paper). https://www.nber. org/papers/w27749

Banca y Negocios (2021). FMI: Latinoamérica no recuperará el nivel previo a la pandemia hasta 2023. Febrero 08, 2021 // EFE. https:// www.bancaynegocios.com/fmi-latinoamerica-no-recuperara-el-nivel-previo-a-la-pandemia-hasta-2023/

Banco Central (2021). Mercado de trabajo Encuesta continua (ENCFT). https://bancentral.gov. do/a/d/2541-encuesta-continua-encft

Barna Management School y COPARDOM (2020). El futuro del mercado del trabajo en la República Dominicana. (Working paper).

Cárdenas, J. y Guataquí, J. (2015). Metodología para el análisis de la demanda laboral mediante datos de internet: El caso colombiano. Revista de Economía del Rosario, 8(1 enero - junio 2015), 93-116. https://www.researchgate.net/ publication/294277142_Metodologia_para_ el_analisis_de_demanda_laboral_mediante_ datos_de_Internet_el_caso_colombiano

Comisión Económica para América Latina y el Caribe -CEPAL. (2020). Los trabajadores de plataformas digitales en la República Dominicana. Caracterización y opciones para su protección social. https://repositorio.cepal.org/bitstream/ handle/11362/45893/1/S2000431_es.pdf

Daxx. (2020a, October 7). Talent Shortage in the Nordics: How Norway, Sweden, Finland, and Denmark Can Solve the Software Developer Shortage in 2021. https://www.daxx.com/ blog/development-trends/nordics-tech-talent-shortage 
Daxx (2020b, October 8). The Software Development Industry in Israel: How Tech Businesses Manage the Tech Talent Shortage. https://www. daxx.com/blog/development-trends/software-development-industry-israel

Deloitte. (2019). Workplace transformation in the digital age. Challenges and success factors. https://www2.deloitte.com/content/dam/ Deloitte/ch/Documents/innovation/ch-enworkplace-of-the-future.pdf

European Commission. (2021). Digital skills and jobs. Policy. https://digital-strategy.ec.europa. $\mathrm{eu} / \mathrm{en} /$ policies/digital-skills-and-jobs

Forbes. (2020). The fastest and slowest growing jobs. https://www.forbes.com/pictures/efkk$45 \mathrm{fmhd} / 7$-customer-service-representatives/\# ab27fa22e 337

Forbes. (2021, January 12,). The Top 25 Soft Skills Remote Workers Need In 2021-And 3 Ways To Get Them. https://www.forbes.com/sites/ markcperna/2021/01/12/the-top-25-softskills-remote-workers-need-in-2021-and-3ways-to-get-them/?sh $=28239961 \mathrm{~d} 78 \mathrm{~b}$

Gartner. (2020, October 19). These trends can help data and analytics leaders navigate their COVID-19 response and recovery and prepare for a post-pandemic reset. https://www. gartner.com/smarterwithgartner/gartner-top10-trends-in-data-and-analytics-for-2020/

Institute for the future of work (2021). The Impact of COVID-19 on Technology Adoption. January 13, 2021. https://www.ifow.org/news-articles/the-impact-of-COVID-19-on-technology-adoption

International Labour Organization (2021). ILO Monitor: COVID-19 and the world of work. Seventh edition Updated estimates and analysis. https://www.ilo.org/wcmsp5/groups/ public/@dgreports/@dcomm/documents/ briefingnote/wcms_767028.pdf

IoD (2020). Home-working here to stay, new IoD figures suggest. https://www.iod.com/news/ news/articles/Home-working-here-to-staynew-IoD-figures-suggest
Korn Ferry (2020a). Future of work. https://www. kornferry.com/challenges/future-of-work

Korn Ferry (2020b). The \$8.5 Trillion Talent Shortage. https://www.kornferry.com/insights/articles/ talent-crunch-future-of-work

La República (2020, 17 de noviembre). Empresas que contraten mayores de 45 años disfrutarian pronto de rebaja en impuesto de la renta. https://www. larepublica.net/noticia/empresas-que-contraten-mayores-de-45-anos-disfrutarian-prontode-rebaja-en-impuesto-de-la-renta

LinkedIn (2021). Jobs on the Rise Reports - The fastest-growing jobs in the world. https://business.linkedin.com/talent-solutions/emerging-jobs-report

Manpower Group (2019a). Se buscan personas: los robots las necesitan. https://www. manpowergroup.com.mx/wps/wcm/ connect/manpowergroup/4b4e 85 e 9 e77d-4226-8b1e-dc8eb968b5c5/MPG_ WEF_SkillsRevolution_4.0_MX-b. pdf?MOD=AJPERES\&CONVERT_TO=ur$1 \&$ CACHEID = ROOTWORKSPACE . Z18_2802IK01OORA70QUFIPQ192 H31-4b4e85e9-e77d-4226-8b1e-dc8eb968b5c5-mAdh0um

Manpower Group. (2019b). Encuesta de escasez de talento 2018. https://www.manpowergroup. com.mx/wps/wcm/connect/manpowergroup/ db65d29b-c8d3-46e9-9af5-fed9ef38a9d0/ MG_EscasezdeTalentoMexico2018.pdf?$\mathrm{MOD}=\mathrm{AJPERES} \& \mathrm{CONVERT} \_\mathrm{TO}=\mathrm{url} \&-$ CACHEID $=$ db65d29b-c8d3-46e9-9af5-fed9ef38a9d0

McKinsey (2019). The future of work in America. People and places, today and tomorrow. https:// www.mckinsey.com/ //media/McKinsey/ Featured $\% 20$ Insights/Future\%20of\%20 Organizations/The $\% 20$ future $\% 20$ of $\% 20$ work $\% 20$ in $\% 20$ America $\% 20$ People $\% 20$ and $\% 20$ places $\% 20$ today $\% 20$ and $\% 20$ tomorrow/MGI-The-Future-of-Work-in-America-Report-July-2019.ashx 
McKinsey (2020). How COVID-19 has pushed companies over the technology tipping point — and transformed business forever. https://www.mckinsey. com/business-functions/strategy-and-corporate-finance/our-insights/how-covid-19-has-pushed-companies-over-the-technology-tipping-point-and-transformed-business-forever

Milasi, S., Bisello, M., Hurley, J., Sostero, M., Fernández-Macías, E. (2020, August). The potential for teleworking in Europe and the risk of a new digital divide. VoxEU - CEPR. https:// voxeu.org/article/potential-teleworking-europe-and-risk-new-digital-divide

Misión Colombia Envejece. (2020). Elmercado laboral $y$ las personas mayores. https://www.saldarriagaconcha.org/wp-content/uploads/2019/01/ pm_mercado_laboral.pdf

Organización Internacional del Trabajo. (2019). Las plataformas digitales y el futuro del trabajo. Cómo fomentar el trabajo decente en el mundo digital. https://www.ilo.org/wcmsp5/groups/ public/---dgreports/---dcomm/---publ/documents/publication/wcms_684183.pdf

Organización Internacional del Trabajo (2020). Nota país. COVID - 19 y el Mundo del Trabajo: Punto de partida, respuesta y desafios en República Dominicana. https://www.ilo. org/wcmsp5/groups/public/---americas/--ro-lima/---sro-san_jose/documents/publication/wcms_755526.pdf

Qlik (2020, November 2). The Top Skills Needed to Succeed in the Fourth Industrial Revolution. https://www.qlik.com/blog/the-top-skillsneeded-to-succeed-in-the-fourth-industrialrevolution
Riom, C. \& Valero, A. (2020, September 30). The London School of economics and political science. [Paper Number CEPCOVID-19-009]. https://cep.lse.ac.uk/_NEW/PUBLICATIONS/abstract.asp?index $=7291$

Salary.com (2020). 7 Best Careers for the Future. https://www.salary.com/articles/7-careersfor-the-future/

Universidad Autónoma de Santo Domingo Facultad de Economía (2020, 18 de octubre). El COVID-19 ha tenido fuerte impacto sobre el empleo en el pais. Domingo. https://www.uasd. edu.do/periodico/index.php/el-universitario/ item/3802-facultad-de-economia-uasd-reitera-que-el-COVID-19-ha-tenido-fuerte-impacto-sobre-el-empleo-en-el-pais

United Nations Industrial Development Organization. (2019). Industrial Development Report 2020. https://www.unido.org/sites/default/ files/files/2019-11/UNIDO_IDR2020-MainReport_overview.pdf

Upwork. (2020). Independent Workforce Report. https://www.upwork.com/documents/independent-workforce-report

Vicepresidencia de la República. (2018). Educación superior dominicana: expansión, desarrollo y perspectivas futuras. Boletin del Observatorio de Politicas Sociales y Desarrollo, 2018https:// www.academia.edu/38464220/Educaci\%C3\%B3n_superior_dominicana_expansi\%C3\%B3n_desarrollo_y_perspectivas_futuras.

World Economic Forum -WEF. (2021). Fourth Industrial Revolution. https://www.weforum. org/focus/fourth-industrial-revolution 